\title{
THE ROLE OF FUNDOPLICATION IN THE TREATMENT OF TYPE II PARAESOPHAGEAL HERNIA
}

Clark B. Fuller, $\mathrm{MD}^{\S}$

Jeffrey A. Hagen, $\mathrm{MD}^{\S}$

Tom R. DeMeester, MD

Jeffrey $\mathrm{H}$. Peters, $\mathrm{MD}^{\S}$

Manfred Ritter, $\mathrm{MD}^{\S}$

Cedric G. Bremner, $\mathrm{MD}^{\S}$
Objectives: The role of fundoplication in patients with pure type II paraesophageal hiatal hernia remains controversial. Conventional thinking suggests that because the lower esophageal sphincter is located within the abdomen, it is competent, and fundoplication is unnecessary. Few studies have used objective evaluation to guide the addition of an antireflux procedure. Methods: Fifteen consecutive patients with type II paraesophageal hernia were treated between May 1991 and July 1994. All had radiographic criteria of pure type II hernias. Preoperative evaluation included upper intestinal endoscopy, esophageal manometry, and 24-hour ambulatory pH monitoring. The lower esophageal sphincter was considered incompetent if any of the following criteria were present: a resting pressure less than $7 \mathrm{~mm} \mathrm{Hg}$, an overall sphincter length less than $2 \mathrm{~cm}$, or an intraabdominal length less than $1 \mathrm{~cm}$. Primary symptoms responsible for surgery were related to the hernia in $73 \%$ of patients: dysphagia or postprandial abdominal pain in six patients, abdominal distension or vomiting in four patients, and bleeding in one patient. Symptoms typical of gastroesophageal reflux were present in four patients: heartburn and regurgitation in two each. Results: Objective evidence of gastroesophageal reflux was present in the majority of patients. Five patients $(31 \%)$ had evidence of esophageal injury: esophagitis in three patients, stricture in one, and esophageal ulcer in one. In 11 of 15 patients (69\%), pathologic esophageal acid exposure was detected by 24-hour pH monitoring. Twelve patients (75\%) had a defective lower esophageal sphincter, usually the result of an inadequate intraabdominal length $(8 / 12,66 \%)$. Hernia reduction, crural closure, and Nissen fundoplication were performed in 14 patients (one patient awaits surgery). Symptomatic relief was excellent in all cases. No patient has had hernia recurrence at an average of 14 months' follow-up (range 2 to 39 months). Conclusion: Objective evaluation reveals that gastroesophageal reflux accompanies type II paraesophageal hernia in a high proportion of patients, usually because of an incompetent lower esophageal sphincter. Appropriate treatment includes reduction of the hernia, crural closure, and fundoplication in most, if not all, patients. (J ThORAC CARDIOvASC SURG 1996;111:655-61)
$A^{\mathrm{p}}$ pure paraesophageal hernia (type II hiatal hernia) is a rare condition characterized by the herniation of variable portions of the stomach into

From the University of Southern California School of Medicine Department of Surgery, Los Angeles, Calif.

Read at the Seventy-fifth Annual Meeting of The American Association for Thoracic Surgery, Boston, Mass., April 23-26, 1995.

Received for publication April 27, 1995; revisions requested Sept. 25, 1995; revisions received Oct. 10, 1995; accepted for publication Nov. 22, 1995.

Address for reprints: Tom R. DeMeester, MD, University of Southern California, Department of Surgery, 1510 San Pablo St., Suite 514, Los Angeles, CA 90033-4612.

${ }^{\S}$ By invitation.

Copyright (C) 1996 by Mosby-Year Book, Inc

$0022-5223 / 96 \$ 5.00+0 \quad \mathbf{1 2 / 6 / 7 0 8 7 6}$ the chest, while the esophagogastric junction remains in its normal position below the diaphragm. The lower esophageal sphincter, by virtue of its normal location, is presumed to be competent. This theory is supported by the clinical observation that reflux systems are uncommon in patients with a paraesophageal hernia. Consequently, surgeons have concluded that studies to detect reflux are not indicated in patients with a paraesophageal hernia and the addition of an antireflux repair is unnecessary.

In contrast, others say that clinical experience indicates just the opposite, that reflux does occur in patients with paraesophageal hernias. The exact prevalence is difficult to determine because in most series preoperative evaluation has been limited. 


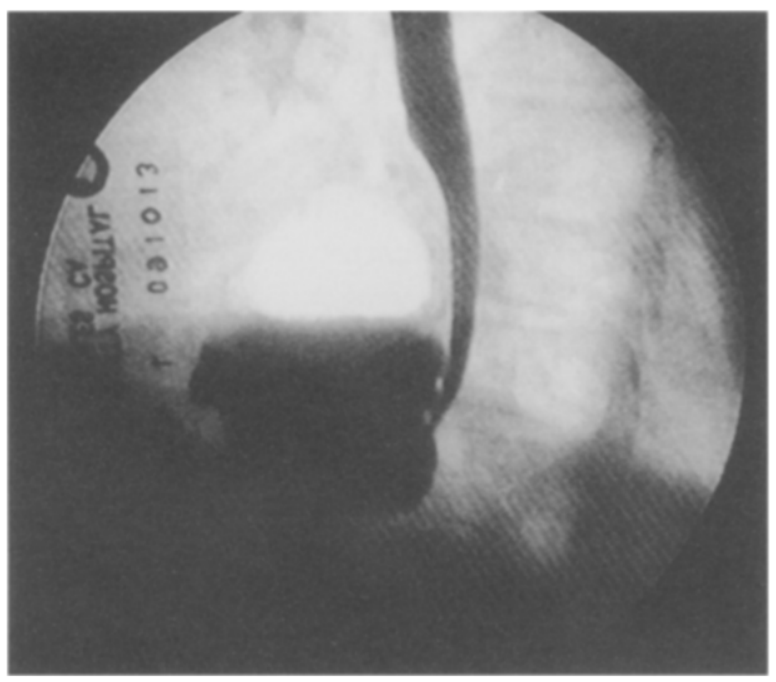

Fig. 1. Lateral projection of a study of the upper gastroesophageal tract demonstrating a pure type II paraesophageal hiatal hernia.

Follow-up studies, however, have shown that up to $65 \%$ of patients not having an antireflux procedure as part of the repair manifest reflux symptoms after the operation. ${ }^{1}$ It is difficult to know whether their symptoms are due to a preexisting defective sphincter or surgical mobilization of the cardia and disruption of the crural attachments during the repair.

This study attempts to resolve these differences by reviewing our experience with the pure paraesophageal hernias. All patients had thorough preoperative testing to define the prevalence of gastroesophageal reflux and determine the mechanisms responsible for it. The goal was to better define the optimal surgical therapy for these patients.

\section{Patients and methods}

Study population. Between May 1991 and July 1994, 393 patients were studied in the foregut function laboratory at the University of Southern California Hospital. Of these, 35 had a paraesophageal hernia, of whom 15 had a pure type II paraesophageal hiatal hernia.

Radiographically, a pure type II hernia was present if the esophagogastric junction was visible below the diaphragm on both supine and upright views, with evidence of herniation of portions of the stomach into the chest. Herniation is best evaluated on a lateral or left posterior oblique view (Fig. 1).

The patients included seven men and eight women, with a median age of 60 years (range 39 to 82 years). Symptoms were objectively scored with the aid of a questionnaire filled out by the laboratory nurse at the time of the initial esophageal evaluation and motility study. The symptoms were classified as being related to the hernia (i.e., dys-
Table I. $p H$ score and endoscopic findings

\begin{tabular}{cccc}
\hline Patient & $\begin{array}{c}\text { pH } \\
\text { score* }\end{array}$ & $\begin{array}{c}\text { Location } \\
\text { of crura } \\
(\mathrm{cm})\end{array}$ & $\begin{array}{c}\text { Location of } \\
\text { GE junction } \\
(\mathrm{cm})\end{array}$ \\
\hline 1 & 34.4 & 32 & 33 \\
2 & 24.9 & 47 & 45 \\
3 & 83.7 & 37 & 46 \\
4 & 20.3 & 40 & 43 \\
5 & 16.5 & - & - \\
6 & 59.8 & 36 & 36 \\
7 & 56.6 & 35 & 35 \\
8 & 55.0 & 40 & 41 \\
9 & 20.2 & 41 & 43 \\
10 & 96.1 & 36 & 37 \\
11 & 20.3 & 37 & 39 \\
12 & 3.7 & 39 & 39 \\
13 & 11.3 & 36 & 37 \\
14 & 0.3 & 32 & 34 \\
15 & 11.7 & 42 & 40
\end{tabular}

$G E$, Gastroesophageal.

*Normal < 14.7.

phagia, postprandial pain, abdominal distention, nausea, vomiting, hematemesis, or anemia) or to gastroesophageal reflux (i.e., heartburn or regurgitation).

Patient investigation. The upper part of the intestine was examined with an endoscope to determine the presence of mucosal injury and to confirm the abdominal position of the gastroesophageal junction, that is, at or distal to the crura (Table I). Mucosal injury was classified as follows: grade I, erythema; grade II, linear erosion; and grade III, cobblestone mucosa, that is, coalescing erosions with islands of epithelium. The presence of a stricture or Barrett's metaplasia, or both, confirmed by the histologic presence of intestinal metaplasia, was as also noted.

Stationary manometry was performed with a single polyvinyl catheter consisting of five fluid-filled tubes with lateral side ports separated by a distance of $5 \mathrm{~cm}$. A stationary pullthrough technique was used to record the lower esophageal sphincter pressure. The resting pressure at the respiratory inversion point, the overall length of the high-pressure zone, and the length exposed to abdominal pressure was measured as previously described. ${ }^{2}$ The mean of the five measurements for each component was recorded. The lower esophageal sphincter was considered defective if one or more of the following were present: a pressure less than $7 \mathrm{~mm} \mathrm{Hg}$, an overall length less than 2 $\mathrm{cm}$, or an abdominal length less than $1 \mathrm{~cm}$. These values have previously been shown to be below the 2.5 percentile of 50 normal subjects. ${ }^{2}$

Twenty-four-hour esophageal $\mathrm{pH}$ monitoring was used to measure esophageal exposure to acid with a glass electrode and a portable solid-state monitor that samples and records esophageal $\mathrm{pH}$ at 6-second intervals. The $\mathrm{pH}$ probe was passed transnasally and positioned $5 \mathrm{~cm}$ above the upper border of the distal esophageal sphincter as measured by manometry. The patient was sent home and instructed to remain in the upright or sitting position until he or she retired for the evening, to avoid strenuous 
Table II. Duration of follow-up

\begin{tabular}{cc}
\hline Years & No. of patients \\
\hline $0-1$ & 8 \\
$1-2$ & 3 \\
$2-3$ & 2 \\
$3+$ & 1
\end{tabular}

Table III. Primary symptoms

\begin{tabular}{ccc}
\hline Symptom & \multicolumn{2}{c}{ Patients } \\
\hline Hernia related & \multicolumn{1}{c}{$11(73 \%)$} \\
Dysphagia/pain & 6 & \\
Distention & 4 & \\
Anemia/bleeding & 1 & \\
Reflux related & \multicolumn{2}{c}{$4(27 \%)$} \\
Heartburn & 2 & \\
Regurgitation & 2 &
\end{tabular}

exertion, and to follow a diet restricted to three meals and composed of food with a pH between 5 and 7 . Only water was permitted between meals. A diary was kept of food and fluid intake, symptoms experienced during the monitored period, the time a supine position was assumed in preparation for sleep, and the time of rising in the morning. At the end of the test the stored data were transferred to an IBM personal computer (IBM Corp., Armonk, N.Y.) and analyzed with a commercial software program (Gastrosoft, Dallas, Tex.).

Acid reflux was indicated by an esophageal $\mathrm{pH}$ of 4 or less. The amount of esophageal acid exposure was scored by means of a previously published mathematical formula based on the mean value and standard deviation for six components of the 24-hour record: percentage of time the esophageal $\mathrm{pH}$ was less than 4 for the total 24-hour period, time in the upright and supine positions, the total number of reflux episodes, the number of episodes lasting 5 minutes or longer, and the duration of the longest episode. Patients whose cumulative score exceeded the mean score of control subjects by more than two standard deviations were considered to have abnormal esophageal exposure to acid gastric juice. ${ }^{3}$

Surgical therapy. Fourteen of the 15 patients have undergone repair of the paraesophageal hernia. The fifteenth is currently waiting for repair. The exposure for the repair was transabdominal in 11 patients and transthoracic in three. All operations were performed electively and all patients had a Nissen fundoplication. Essential components of the procedure included reduction of the hernia, excision of the hernia sac, crural approximation, and construction of the fundoplication. ${ }^{4}$

Follow-up. All patients were observed, with a mean follow-up of 14 months. Specific length of follow-up is shown in Table II. Changes in the patient's initial symptoms were recorded and the emergence of new symptoms was identified.

Statistical methods. Proportions between groups were compared by Fisher's exact test. The Student's $t$ test was used for comparison of means. A $p$ value of less than 0.05 was considered significant.
Table IV. Findings of 24-hour esophageal pH monitoring

\begin{tabular}{|c|c|c|}
\hline \multirow[b]{2}{*}{ Component } & \multicolumn{2}{|c|}{$\begin{array}{l}\text { Patients } \\
(n=15)\end{array}$} \\
\hline & No. & $\%$ \\
\hline Abnormal No. of reflux episodes & 9 & 60 \\
\hline Abnormal No. of episodes $>5 \mathrm{~min}$ & 8 & 53 \\
\hline Abnormally prolonged reflux episode & 2 & 13 \\
\hline Abnormal percentage of time $\mathrm{pH}<4$ & 6 & 40 \\
\hline Upright position & 6 & 40 \\
\hline Supine position & 10 & 67 \\
\hline
\end{tabular}

\section{Results}

The primary symptoms are listed in Table III. Hernia-related symptoms were present in $73 \%$ and symptoms of reflux were predominant in $27 \%$.

Endoscopic examination revealed evidence of mucosal injury in the esophagus of five patients (31\%): three with grade 2 esophagitis, one with grade 3 esophagitis, and one with a stricture.

Increased esophageal exposure to acid was present in 11 patients $(69 \%)$. The total number of reflux episodes was abnormal in nine of 11 , and percentage of time that $\mathrm{pH}$ was less than 4 in the supine period was abnormal in 10. Abnormally prolonged reflux episodes were seen in only two patients and, in these, prolonged reflux was not an isolated finding (Table IV).

Esophageal manometry revealed a mechanically defective lower esophageal sphincter in 12 patients $(75 \%)$. Half had more than one defective parameter. Defects in resting pressure or abdominal length were present in three fourths of the patients. All patients had normal esophageal body function.

Three patients had a mechanically normal lower esophageal sphincter. All had normal esophageal acid exposure. Of the 12 patients who had a defective sphincter, all but one had increased esophageal acid exposure. The remaining patient had a mechanically defective sphincter without increased acid exposure in the esophagus.

The average length of stay in the hospital was 6.3 days. For the patients who underwent laparoscopic Nissen procedures, the mean length of stay was 3.6 days, whereas patients stayed an average of 7.7 days after an open procedure $(p<0.001$; Student's $t$ test $)$. No postoperative complications were encountered.

At follow-up, all patients reported that their specific preoperative symptoms had been relieved and none had new symptoms. Specifically, all patients were free of dysphagia. 


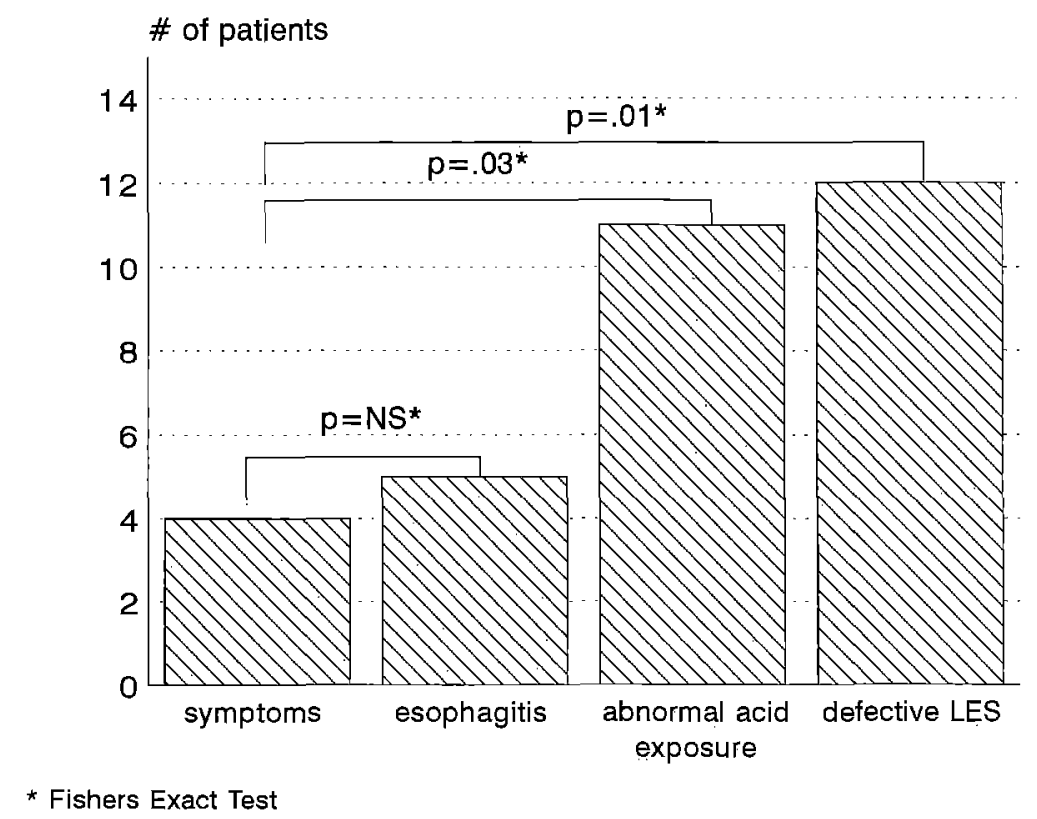

Fig. 2. Comparison of the prevalence of gastroesophageal reflux on the basis of symptoms and by objective studies. LES, Lower esophageal sphincter; NS, not significant.

\section{Discussion}

Paraesophageal hernias should be repaired when they become apparent, even if minimally symptomatic, because of their propensity to produce the life-threatening complications of incarceration, ischemia, and perforation. ${ }^{5}$ Controversy exists with regard to adding a fundoplication to the repair. Central to this disagreement is a difference of opinion as to the prevalence of gastroesophageal reflux in these patients.

In the past, surgeons have relied heavily on the presence of symptoms as an indicator of reflux. There are problems with this approach. Our study has shown that symptoms are unreliable indicators of reflux, because increased esophageal acid exposure was measured in $69 \%$ of the patients whereas symptoms of reflux were present in less than $30 \%$. Some patients may have had few reflux symptoms because of the compressive effects of the large anterior hernia sac, containing the fundus of the stomach adjacent to the distal esophagus. Our study showed that $75 \%$ of the patients had mechanically defective sphincters, yet only $30 \%$ had reflux symptoms, supporting the potential protective role of the compressive hernia sac and reduction of the fundus of the stomach, without correction of the defective sphincter, could unmask gastroesophageal reflux.
This may explain the observation by Treacy and Jamieson $^{1}$ that reflux symptoms occurred after repair without fundoplication in $65 \%$ of patients, compared with $21 \%$ when fundoplication was added.

A similar argument can be made for relying, as some authors have advocated, on endoscopic findings as an indication of reflux. ${ }^{6}$ Although endoscopic esophagitis is more specific for reflux disease, the absence of esophagitis does not preclude the presence of significant reflux. ${ }^{7,8}$ This finding was confirmed in our series. Although marginally more sensitive than symptoms alone (31\% vs $27 \%$ ), endoscopic examination still failed to detect pathologic reflux in seven of the 12 patients who had objective evidence of this disorder.

Some authors have suggested that because the lower esophageal sphincter is in the normal position, by definition, reflux never occurs ${ }^{9}$ and testing is therefore unnecessary. In our study 24 -hour $\mathrm{pH}$ studies showed objective evidence of pathologic reflux in nearly three quarters of patients with paraesophageal hernias. This figure is similar to the prevalence of reflux demonstrated by Walther and associates, ${ }^{10}$ documenting again the presence of reflux in most of these patients despite the absence of symptoms. Other authors have suggested that 
testing be performed only in patients with symptoms. Our data suggest that this approach would fail to detect pathologic reflux in the majority of patients who would benefit from fundoplication (Fig. 2).

The addition of the fundoplication may not be necessary in some patients, such as those who are free of reflux symptoms and who have normal esophageal acid exposure and a mechanically normal lower esophageal sphincter. In our experience, $25 \%$ of patients with paraesophageal hernias can be so categorized. If excessive dissection of the crural region is avoided, an antireflux repair could potentially be omitted.

Our results also have implications for the management of patients who require emergency treatment. In this setting, with little objective information available, symptoms are often used to determine whether fundoplication should be performed. Our data suggest that a safer course is to assume that reflux is present, rather than rely on symptoms, and to add a fundoplication to the repair.

On the basis of this experience, we conclude that the approach to patients with paraesophageal hernias should include routine use of complete esophageal function studies to determine with certainty whether pathologic reflux is present. When pathologic reflux is present, a fundoplication must be included in the repair. In urgent situations in which the patient cannot be studied before the operation, such as in the setting of acute volvulus, adding a fundoplication to the repair is recommended. In the unusual patient who has a mechanically normal sphincter and normal esophageal acid exposure, a simple reduction of the hernia and closure of the crural defect can be done.

\section{REFERENCES}

1. Treacy PJ, Jamieson GG. An approach to the management of para-oesophageal hiatus hernias. Aust N Z J Surg 1987;57: 813-7.

2. Zaninotto G, DeMeester TR, Schwizer W, Johansson K, Chung SC. Lower esophageal sphincter in health and disease. Am J Surg 1988;155:104-11.

3. Jamieson JR, Stein HJ, DeMeester TR, et al. Ambulatory 24-hour esophageal $\mathrm{pH}$ monitoring: normal values, optimal thresholds, specificity, sensitivity, and reproducibility. Am J Gastroenterol 1992;87:1102-11.

4. DeMeester TR, Bonavina L, Albertucci M. Nissen fundoplication for gastroesophageal reflux disease-evaluation of primary repair in 100 consecutive patients. Ann Surg 1986; 204:9-20.

5. Skinner DB, Belsey RH. Surgical management of esophageal reflux and hiatus hernia: long-term results with 1,030 patients. J Thorac Cardiovasc Surg 1967;53:33-54.
6. Williamson WA, Ellis FH Jr, Streitz JM, Shahran DM. Paraesophageal hiatal hernia: Is an antireflux procedure necessary? Am Thorac Surg 1993;56:447-52.

7. Ismail-Tsergi F, Horton PF, Pope CE. Histologic consequences of gastroesophageal reflux in man. Gastroenterology 1970;S8:163-24.

8. Ismail-Tsergi F, Pope CE. Distribution of the histologic changes of gastroesophageal reflux in the distal esophagus in man. Gastroenterology 1974;66:1109-13.

9. Payne WS. Paraesophageal hiatal hernia. In: Nyhus LM, Baker RJ, eds. Mastery of surgery. Boston: Little Brown, 1992;420-8.

10. Walther BS, DeMeester TR, Lafontaine E, Courtney JV, Little AG, Skinner DB. Effect of paraesophageal hernia or sphincter function and its implication on surgical therapy. Am J Surg 1984;147:111-6.

\section{Discussion}

Dr. Victor F. Trastek (Rochester, Minn.). The addition of an antireflux procedure to the repair of a type II or pure paraesophageal hernia affects a very small subgroup of patients. Despite this, the issue remains alive and controversial, and you will find experienced surgeons on both sides of the fence.

There are probably two reasons to consider adding fundoplication to the repair of the hernia. One is that the patients have preoperative reflux disease. Dr. Fuller and his colleagues have nicely shown that reflux occurs in a larger group of patients than we may have thought. The continued emphasis by you and your group on thorough preoperative evaluation helps to make this clear. The second reason for adding fundoplication would be if the type of repair you perform requires full mobilization of the gastroesophageal junction such that you may cause reflux to occur; therefore an antireflux procedure would be indicated to keep that from occurring.

We have continued to add the antireflux procedure, but probably more for the second reason than the first. I believe this information does reinforce this approach.

I have three questions. In your manuscript, you supplied no information on the morbidity of the operation. When we promote a more complex solution to a problem, we have to weigh the risk versus the benefits. Thus I would ask about the morbidity of your operation.

Second, the operation was successful in all 14 patients in that none had symptoms, yet no postoperative objective data were presented, showing that the antireflux procedure was successful. Inasmuch as your premise is that if we study these patients, we will find that many have reflux and therefore we ought to do the procedure, then postoperative objective data would be helpful also. Did you have any objective data from the postoperative period?

Third, 11 of your 14 patients had abdominal operations. What surgical technique are you recommending for the treatment of this particular problem? In particular, are you using the laparoscopic Nissen fundoplication?

Dr. Hagen. I will answer the third question first. Because we accomplished a Nissen through the abdomen in most of these patients and we have been using laparoscopic Nissen procedures, even in patients with paraesophageal hernias, we wanted to make sure that we were 
doing the right thing including an antireflux repair. We are currently using a laparoscopic approach in most of these patients.

There was no major morbidity in the series of 15 patients, to a large extent, I think, because approximately half of the patients procedures were done laparoscopically. Morbidity has been minimal, and the hospital stays have been short.

You inquired about objective documentation of the results after the operation. We do not have any, and that is obviously an area to be pursued.

Dr. Warren A. Williamson (Burlington, Mass.). I would like to ask three questions based on three observations that we have made with a similar study of 20 patients. We have used both preoperative and postoperative esophageal manometry and 24-hour $\mathrm{pH}$ studies in 20 patients with paraesophageal hiatal hernias. There are 11 patients with type II hernias in whom the follow-up is complete at this time. None of these 11 patients had manometric evidence of a hypotensive or shortened lower esophageal sphincter; in fact, the sphincter length was at least $4 \mathrm{~cm}$ in all of these patients. Two of the patients had preoperative evidence of incomplete relaxation of the lower esophageal sphincter, and values returned to normal after anatomic repair.

How accurate is esophageal manometry in patients with paraesophageal hiatal hernia? Does a large paraesophageal defect, with an intrathoracic stomach that is sometimes distended and obstructed and other times decompressed, impair our ability to measure accurately pressures in the lower esophageal sphincture?

My second question relates to the observation that most of these patients have an obstruction of some sort. They have postprandial pain, bloating, vomiting, and dysphagia, which are all obstructive symptoms. How accurate are 24-hour $\mathrm{pH}$ studies in the presence of obstruction? Obstruction will render a competent sphincter incompetent, and thus the presence of acid reflux in many of these patients may simply be a function of obstruction rather than a defective lower esophageal sphincter.

My third question relates to your conclusions. Because no 24-hour $\mathrm{pH}$ or manometric studies were performed in any of your patients after the operation, what objective evidence do you have that adding a fundoplication corrected the hypotensive lower esophageal sphincter or abnormal $\mathrm{pH}$ studies in these patients?

Dr. Hagen. With respect to the final question, it would be nice to have postoperative studies on all these patients. It would be important to confirming, again, the benefits of the Nissen fundoplication. We don't have that information available, unfortunately. However, I see no reason to believe that the results should be any less than the excellent results seen after the Nissen fundoplication for the typical hiatal hernias.

You inquired about the accuracy of manometry in these patients with large paraesophageal hernias. I would speculate that a component of compression or obstruction or restriction at the hiatus caused by the hernia would falsely elevate the pressures measured at the lower esophageal sphincter. If the sphincter is demonstrated to be mechanically defective in the presence of additional tissue or compressive effects, I suspect it would be at least as defective when that tissue was removed.

In answer to your question regarding the accuracy of the $\mathrm{pH}$ studies, the most common abnormality was an abnormal number of reflux episodes, which I think is relatively independent of the effects of the hernia itself. Some of the other factors, for instance, the number of episodes that are longer than 5 minutes, could be affected by clearance of the esophagus. Clearly, the duration of the longest reflux episode could be expected to be abnormal on the basis of obstruction, but these were uncommon. Again, the most common finding was an abnormal number of reflux episodes, leading us to think that it is a reliable measure, independent of esophageal obstruction in these patients.

Dr. Rodney J. Landreneau (Pittsburgh, P.a.). I disagree with your conclusions that fundoplication is necessary after the repair of a type II paraesophageal hernia. It has not been our experience that you add anything but the potential for postfundoplication postoperative complications in the majority of patients in these circumstances.

The biggest issue, though, is the one that Dr. Trastek brought up in asking how these problems were approached, the majority being done through the abdomen and very few through the chest. I implore surgeons who are board-certified in thoracic surgery and have an interest in esophageal surgery to be prepared to go below the diaphragm and not be restricted by this flap of muscle in dealing with paraesophageal herniation. I believe that for type II paraesophageal hernias the abdominal approach is preferred.

I also agree with Dr. DeMeester and his general surgical colleagues that careful manometric studies of these patients are in order; however, their data are in question. The biggest problem that we had manometrically in this group was with the overall intraabdominal length. The other parameters, as Dr. Williamson mentioned, were not abnormal. That in combination with mechanical obstruction creates a real problem in trying to draw conclusions from the manometric data in this setting.

We think that the best approach is to evaluate the patient endoscopically. If the patient has symptoms of reflux and clinical esophagitis and if the risk is low, then the additional fundoplication is warranted.

With the thoracic approach, as Dr. Trastek also said, fundoplication is necessary as a form of pexation, if nothing else. And if the abdominal approach is used there are other methods that can be applied.

Dr. Arthur N. Thomas (San Francisco, Calif.). The conclusion implies that the only treatment for reflux is the Nissen fundoplication. Quite a few people, including Dr. Hill and me, believe that posterior gastropexy and other forms of antireflux procedure are as effective as a Nissen fundoplication, or more effective, in my opinion. I would have been happier if the authors had concluded that an antireflux procedure or fixation procedure was warranted rather than that everybody gets a Nissen fundoplication regardless of the reflux procedure used.

Dr. Hagen. I accept your criticism of endorsing a particular type of operation, namely the Nissen fundoplication. In the manuscript we addressed this issue in more 
detail. In addition to characterizing the sphincter manometrically, we found that all of these patients had normal esophageal motility. It is our contention that a complete fundoplication confers the best protection against reflux. As a result, it is our preference in patients with normal motility.

Dr. John R. Benfield (Sacramento, Calif.). Within recent weeks I had occasion to revise by transthoracic approach two Nissen fundoplications in referred patients who had significant dysphagia from their procedures. This prompts me to make some spontaneous remarks.

A fundamental goal of antireflux operations is to assure that the gastroesophageal junction is below the diaphragm. We have just heard a proposal to add a fundoplication to repair of paraesophageal hernia-a condition in which the gastroesophageal junction is in normal location below the diaphragm. I respect the group that has made this presentation; however, the information presented has not convinced me to change from the belief that one needs to focus on repairing the primary paraesophageal hernia, and that the addition of the fundoplication may exposes the patient to a risk of complications from a procedure that does not address the primary defect for which one is operating.

Dr. Clement Hiebert (Portland, Maine). Correction of a hiatal hernia of any name has several components: reducing the hernia, narrowing the escape route, and tethering it, just in case. Depending on which technique you use, antireflux sutures require three to six more sutures. The argument for taking these sutures is compelling-the difficulty of redo operations in this region and the impossibility of knowing if competency of the cardia in a massive hernia translates into competency when the stomach is reduced.

Dr. Hagen. I would like to add to that comment. I think that when most surgeons repair a paraesophageal hernia, they recognize the importance of some sort of fixation of the stomach within the abdomen. When you are approaching these transabdominally, you have the choice of a gastropexy, gastrostomy, or fundoplication. Because we can document evidence of the potential for, if not symptoms of, gastroesophageal reflux in these patients, we believe that the best method of fixing the stomach within the abdomen is a fundoplication. 